\title{
Monitoring of Space and Earth electromagnetic environment by MAGDAS project: Collaboration with IKIR - Introduction to ICSWSE/MAGDAS project
}

\author{
Akimasa Yoshikawa ${ }^{1, *}$, Akiko Fujimoto ${ }^{1}$, Akihiro Ikeda ${ }^{1}$, Teiji Uozumi $^{1}$, and Shuji Abe ${ }^{1}$ \\ International Center for Space Weather Science and Education, Kyushu University, Japan
}

\begin{abstract}
For study of coupling processes in the Solar-Terrestrial System, International Center for Space Weather Science and Education (ICSWSE), Kyushu University has developed a real time magnetic data acquisition system (the MAGDAS project) around the world. The number of observational sites is increasing every year with the collaboration of host countries. Now at this time, the MAGDAS Project has installed 78 real time magnetometers - so it is the largest magnetometer array in the world. The history of global observation at Kyushu University is over 30 years and number of developed observational sites is over 140. Especially, Collaboration between IKIR is extended back to 1990's. Now a time, we are operating Flux-gate magnetometer and FM-CW Radar. It is one of most important collaboration for space weather monitoring. By using MAGDAS data, ICSWSE produces many types of space weather index, such as EEindex (for monitoring long tern and shot term variation of equatorial electrojet), Pc5 index (for monitoring solar-wind velocity and high energy electron flux), Sq-index (for monitoring global change of ionospheric low and middle latitudinal current system), and Pc3 index (for monitoring of plasma density variation at low latitudes). In this report, we will introduce recent development of MAGDAS/ICSWSE Indexes project and topics for new open policy for MAGDAS data will be also discussed.
\end{abstract}

\section{What is ICSWSE?}

As a strategy of enhancement and sustainability of our project, Kyushu university reorganized the Space Environment Research Center (SERC) of Kyushu University was re-organized on 01 April 2012. On that date it became the "International Center for Space Weather Science and Education (ICSWSE) ". To allow for easy pronunciation, the short name of the Center is "iku-sei", which means "to nurture" in Japanese.

The purpose for this re-organization is to allow space weather research to continue on a more global basis, and to establish a permanent international institution (center) for space weather science and education. This new center was also specifically requested as a part of the International Space Weather Initiative (ISWI) in the "Abuja ISWI Resolu-

\footnotetext{
Corresponding author: yoshi@geo.kyushu-u.ac.jp
} 
tion" which was unanimously approved by the participants of the "UN/Nigeria Work shop on ISWI" (Abuja, Nigeria, 2011).

The purposes of ICSWSE are:

- expansion and promotion of Space Weather as a field of science;

- closer investigation of changes of climate/geospace/disasters;

- examination of the medical and biological aspects of man working in space;

- realizing international space weather research and education.

The new center is well-placed to achieve these purposes because of the strong human network created by the MAGDAS Project and its associated Magnetometer Data Archives (http://www.icswse.kyushu-u.ac.jp/data/index.php

There are various research activities taking place in ICSWSE. The ICSWSE is composed of 6 research divisions: Geospace atmosphere coupling lab, Geospace fundamental theory lab, Geospace hazard simulation lab and Space medicine lab. The new ICSWSE will engage in the following: Enlarge the scope of its space weather research; evolve internationally as the Center for Space Weather Science and Education; conduct more detailed investigation of the links between geospace changes, climate changes, and natural disasters; start new research in the biomedical aspects of geospace.

\section{The $\mathbf{3 0}$ years history of geomagnetic observations by Kyushu University}

For space environment monitoring, for this 30 years, ICSWSE has developed a real time magnetic data acquisition system (the MAGDAS project) around the world. The number of observational sites is increasing every year with the collaboration of MAGDAS host countries. Now at this time, the MAGDAS Project has installed 78 real time magnetometers - so it is the largest magnetometer array in the world.

Here we introduced 30 years history of geomagnetic observations by Kyushu University.

The Equatorial Magnetometer Network (EMPN) project was started in 1985 and continued to 1995 at the Kyushu University. The P-I of this project is Prof. T.-I. Kitamura. This network had total of 44 stations, especially along the magnetic equator. In this project, we developed and manufactured the magnetometer system ourselves. Researchers stayed observation site for a few months, and took care the system, then brought back data with us.

While the 210 Magnetic Meridian (MM) magnetic observations project started in 1990 and continued to 1995 at Nagoya University. The P-I of this project was first Prof. M. Tanaka and it taken over by Prof. K. Yumoto. Stations densely distributed along 210MM and this project becomes the foundation of collaboration between Russian academy and Japan. This network had total of 34 stations.

The Circum-pan Pacific Magnetic Network (CPMN) Project was started in 1996. The EMN and 210MM were integrated into the CPMN by Prof. Yumoto at Kyushu University. After that, some other stations were appended. Total of 93 stations belong this network.

Real-time monitoring of geomagnetic field, the MAGDAS/CPMN Project, started in 2005. Now at this time, the MAGDAS Project has installed 78 real time magnetometers and total of 140 stations were installed all over the world including non-real time stations. The P-I of this project is now taken over by Prof. A, Yoshikawa. 


\section{Capacity building activity in ICSWSE}

The installation and maintenance of MAGDAS is strongly supported by the cooperation of local collaborators. Many students as well as foreign partners attended field works. This MAGDAS activity has become the foundation of our capacity building activities.

Consistent with the goals of the ISWI, the ultimate goal of our Core-to-Core research program is to:

- strengthen the space-weather-related research abilities of young scientists in the Asia region and Africa region;

- expose young Japanese scientists to more internationally-oriented science activities, such as doing field work in Asia and Africa.

The primary agenda, therefore, is to conduct Capacity Building for young scientists in Japan and Asia/Africa regions through a wide-variety of approaches:

- conducting overseas ISWI/MAGDAS Schools;

- implementing student exchanges;

- installing MAGDAS in "missing areas";

- bringing Asians and Africans to study at Japanese universities.

Through this myriad of approaches, a human network for space weather research is established.

With the support of JSPS, one of the capacity building activities we have at ICSWSE is through trainings. We invite our MAGDAS hosts for training at ICSWSE. We have conducted four trainings so far. Our trainees were from the Philippines, Malaysia, Indonesia Vietnam, Peru, Ivory Coast, Mozambique, Nigeria and Egypt. We teach our trainees how to install and maintain our magnetometers in their respective countries. We also teach them how to monitor how to use a data for their research. At the end of the MAGDAS training, we awarded each of the trainees a certificate of completion. For more information, access the Research Note, Space Research Today, Volume 188, December 2013, Pages 18-20, ISSN 1752-9298, http://dx.doi.org/10.1016/ j.srt.2013. 11.007. (http://www.sciencedirect.com/science/article/pii/S1752929813000601)

We also organized international MAGDAS School with collaboration of UN/OOSA, ISWI, COSPAR, and SCOSTEP. The schools were held at Egypt (2009), Nigeria (2011), Indonesia (2012), Cote d'Ivoire (2013), Peru, Malaysia, Indonesia (2014), Japan (Fukuoka: 2015) and Nigeria, Russia, Peru, Malaysia (2016).

The topics is the UN/JAPAN Space Weather Workshop held at Fukuoka, Japan 20015 March. In this workshop, participants were 160 people from 32 countries and 9-science session and 4 panel discussions were organized. The recommendations and observations of this workshop were officially reported at UN COUPUS and UN general assembly.

We finally repot an education through MAGDAS project in Kyushu University. We dispatched over 80 graduate students to over sea fieldwork during 2010 to 2016. After 1996, 19 Japanese students received Ph.D and after 2012, 6 foreign student received Ph.D through MAGDAS project. They have strong relationship to ICSWSE activity through Offering collaboration, Convening of International MAGDAS workshop in each country, and Student exchange and Researcher exchange. 


\section{Research activity of MAGDAS project in ICSWSE and collaboration with IKIR}

Geomagnetic field variations on the ground are generated by reflecting: "solar terrestrial coupling" electromagnetic disturbances across the magnetosphere and ionosphere, "horizontal coupling" electromagnetic energy is distributed from polar to equatorial ionosphere and vice verse and "atmospheric vertical coupling" propagation of lower atmospheric disturbances to upper atmosphere via gravity wave and tidal waves. By producing space index by using geomagnetic field variations, we can investigate influences to Earth system caused by solar eruptions, relation between solar activity and climate phenomena and/or climate change, global circulation of energy and material of upper atmosphere, and relations between seismic activity and solar activity. Super Multipoint Geomagnetic field Network Observationimportant tool for exploring of frontier of solar terrestrial coupling process. By using MAGDAS data, ICSWSE produces many types of space weather index, such as EE-index (for monitoring long tern and shot term variation of equatorial electrojet), Pc5 index (for monitoring solar-wind velocity and high energy electron flux), Sq-index (for monitoring global change of ionospheric low and middle latitudinal current system), and Pc3 index (for monitoring of plasma density variation at low latitudes). Since 2012, MAGDAS project produced 56 papers in the international refereed Journal.

Collaboration between IKIR is extended back to 1990's. Now a time, we are operating Flux-gate magnetometer and FM-CW Radar. It is one of most important collaboration for space weather monitoring. In June 2017, we visited IKIR and fixed the problem of FM-CW Radar system, and the data successfully begun to obtain. We now plan to measurement of Poynting flux by using magnetic field data obtained MAGDAS and electric field data from FM-CW radar. By developing this new type of Poynting flux measurement system, we expected to understand global circulation of electromagnetic energy and materials through ionosphere. So the collaboration between IKIR and ICSWSE become key element for succession of this new trial. 